\title{
Carcinoma del glomus carotideo. Serie de casos
}

\author{
Carcinoma of the carotid glomus. Case series \\ Andrés Chala-Galindo' (D), Andrea Carolina Albornoz-Garzón² (D, \\ Carlos Eduardo Gómez-Vera ${ }^{3}$
}

1 Médico, especialista en Cirugia General y Cirugia de cabeza y cuello y cirugía reconstructiva microvascular; profesor titular, Departamento Quirúrgico, Universidad de Caldas. Jefe del Servicio de cirugía de cabeza y cuello, Oncólogos de Occidente, Manizales, Colombia.

2 Médico Interno, Universidad de Caldas, Manizales, Colombia.

3 Médico, especialista en Cirugía general y Cirugía vascular periférica, Clínica Avidanti; profesor asociado titular, Departamento Quirúrgico, Universidad de Caldas, Manizales, Colombia.

\section{Resumen}

Introducción. El carcinoma del glomus carotideo es infrecuente y se presenta en el $6 \%$ de los paragangliomas carotideos. Suele haber dificultad en su diagnóstico preoperatorio y no se diferencia fácilmente de su contraparte benigna, lo que puede conllevar a complicaciones quirúrgicas. Este trabajo describe la experiencia en su abordaje clínico y quirúrgico en una serie de casos, además de una revisión de la literatura profundizando en su diagnóstico, abordaje terapéutico, sobrevida y mortalidad.

Métodos. De los pacientes operados por glomus carotideos durante un periodo de 20 años, se identificaron aquellos llevados a cirugía por carcinoma. Se realizó una búsqueda bibliográfica extensa en PubMed, haciendo énfasis en diagnóstico y tratamiento.

Resultados. De un total de 139 pacientes sometidos a cirugía de resección de tumor del cuerpo carotideo, tres pacientes $(2,2 \%)$ presentaron carcinoma. Dos tenían metástasis cervicales y uno metástasis hepáticas. Uno presentaba glomus bilateral asociado a trastorno genético de origen familiar. Se realizó cirugía y adyuvancia en todos los casos.

Discusión. El diagnóstico diferencial preoperatorio entre glomus benigno y maligno es difícil, por lo cual deben buscarse hallazgos clínicos o factores de riesgo que puedan sugerir malignidad. Las imágenes pocas veces muestran características claras de invasión vascular. Siempre que sea posible, en el tratamiento del cáncer del glomus carotideo, debe realizarse resección quirúrgica, acompañada de un vaciamiento funcional de cuello, dejando la radioterapia, la quimioterapia y la hormonoterapia como terapias adyuvantes.

Palabras clave: glomus carotideo; paraganglioma; tumor; cáncer; resección quirúrgica.

Fecha de recibido: 9/08/2020 - Fecha de aceptación: 17/08/2020 - Fecha de publicación en línea: 05/03/2021

Correspondencia: Andrés Chala Galindo, Calle 24 \# 39 - 57, Manizales, Colombia. Teléfono: 3155401920

Correo electrónico: andreschala@hotmail.com

Citar como: Chala-Galindo A, Albornoz-Garzón AC, Gómez-Vera CE. Carcinoma del Glomus carotideo. Serie de casos. Rev Colomb Rev Colomb Cir. 2021;36:257-67. https://doi.org/10.30944/20117582.743

Este es un artículo de acceso abierto bajo una Licencia Creative Commons - BY-NC-ND https://creativecommons.org/licenses/by-ncnd/4.0/deed.es 


\begin{abstract}
Introduction. Carcinoma of the carotid glomus is rare and occurs in 6\% of carotid paragangliomas. There is often difficulty in its preoperative diagnosis, and it is not easily differentiated from its benign counterpart, which can lead to surgical complications. This study describes the experience in its clinical and surgical approach in a series of cases, as well as a review of the literature of its diagnosis, therapeutic approach, survival, and mortality.

Methods. Of the patients operated for carotid glomus over a 20-year period, those undergoing surgery for carcinoma were identified. An extensive literature search was conducted in PubMed, with an emphasis on diagnosis and treatment.

Results. Out of a total of 139 patients who underwent carotid body tumor resection surgery, three patients (2.2\%) had carcinoma. Two had cervical metastases and one had liver metastases. One had bilateral glomus associated with a genetic disorder of familial origin. Surgery and adjuvant surgery were performed in all cases.

Discussion. The preoperative differential diagnosis between benign and malignant glomus is difficult, for which clinical findings or risk factors that may suggest malignancy should be sought. Images rarely show clear features of vascular invasion. Whenever possible in the treatment of carotid glomus cancer, surgical resection should be performed, accompanied by a functional neck dissection, leaving radiotherapy, chemotherapy, and hormone therapy as adjuvant therapies.
\end{abstract}

Keywords: carotid glomus; paraganglioma; tumor, cancer; surgical resection.

\section{Introducción}

El glomus carotideo, paraganglioma del cuerpo carotideo o tumor del cuerpo carotideo, es una entidad neoplásica, usualmente benigna, que hace parte de los paragangliomas extra-adrenales, siendo entre estos, el más frecuente. Se origina en los quimiorreceptores del cuerpo carotideo, los cuales se ubican en la adventicia, por lo cual también recibe el nombre de quemodectoma. La mayor incidencia del glomus carotideo se observa en poblaciones que se encuentran a más de 1000 metros sobre el nivel del mar.

Se cree que estos tumores se generan debido a una respuesta a la hipoxia crónica por parte de las células tipo 1, también llamadas células glómicas, causando en primer lugar hipertrofia y finalmente hiperplasia ${ }^{1}$. En algunos mamíferos se ha descrito que la inactivación de la prolil-hidroxilasa PHD2, que es el principal regulador del factor inducido por hipoxia (HIF-2 $\alpha$ ), produce la expansión del cuerpo carotideo, característica del glomus carotideo $^{2}$.
La presentación maligna del glomus carotideo es rara, describiéndose en la literatura diferentes prevalencias que varían entre el $6 \%$ y el $20 \%$, oscilando en un promedio del $10 \%$ al $12 \%{ }^{3,4}$. Entre los paragangliomas de cabeza y cuello, el del cuerpo carotideo presenta la menor tasa de malignidad, con incidencia de metástasis del $1,4 \%{ }^{5}$. Desde 1971 se ha reportado la dificultad en el diagnóstico de malignidad del glomus carotideo y en el establecimiento del riesgo quirúrgico, para lo cual Shamblin y colaboradores propusieron una clasificación basada en la relación macroscópica tumor-vaso, dividiéndolos en tres grupos ${ }^{6}$.

En este trabajo se describe la experiencia del abordaje quirúrgico del glomus carotideo maligno, en un periodo de 20 años, en el servicio de Cirugía de Cabeza y Cuello de la Universidad de Caldas, y se realiza una revisión de la literatura enfatizando en la dificultad del diagnóstico, el abordaje terapéutico, la sobrevida y la mortalidad de estos pacientes. 


\section{Métodos}

Se revisó la base de datos de los pacientes sometidos a cirugía con diagnóstico de tumor del glomus carotideo, en el servicio de Cirugía de Cabeza y Cuello, entre el 1 de enero de 1999 y el 31 de diciembre de 2018. Se analizó su diagnóstico, tratamiento y sobrevida. Se realizó una búsqueda bibliográfica extensa en PubMed utilizando la fórmula de búsqueda (C"Paraganglioma, Extra-Adrenal"[Mesh]) AND "Neoplasms"[Mesh]) OR "Mixed Tumor, Malignant"[Mesh]))

\section{Resultados}

De un total de 139 pacientes registrados en la base de datos del servicio de Cirugía de Cabeza y Cuello sometidos a cirugía de resección de tumor del cuerpo carotideo, tres pacientes $(2,2 \%)$, dos mujeres y un hombre, con edades entre los 35 y 61 años, fueron diagnosticados con carcinoma del glomus carotideo. El diagnóstico preoperatorio fue realizado solo en un caso. Dos se presentaron con metástasis cervicales y uno, adicionalmente, con metástasis hepáticas. En un paciente se identificó glomus bilateral, con historia de presentación bilateral en cinco miembros de su familia. Se practicó cirugía y adyuvancia en todos los casos. Todos los pacientes sobreviven hasta la fecha y están en seguimiento activo, uno de ellos aún presenta actividad tumoral persistente estable. Se describe cada caso a continuación.

\section{Caso 1}

Mujer de 35 años, consultó por la presencia de masa cervical bilateral, de tres años de evolución, localizada en el espacio carotideo, asintomática, con mínimo crecimiento de la lesión desde su aparición. Dos hermanos y una hermana tenían masas similares y una sobrina un glomus bilateral sin malignidad. El examen físico de la cavidad y la orofaringe fue normal y el examen del cuello reveló la presencia de dos masas cauchosas, cada una en la zona de la bifurcación carotidea, compresibles y con signos de Chevassu y Fontaine presentes, a la auscultación con soplo holo-sistólico sincrónico al pulso, hallazgos clínicos sugestivos de glomus carotideo bilateral.
Se realizó arteriografía selectiva que documentó la presencia de glomus carotideo bilateral. Se programó una resección de estos en dos tiempos, iniciando por la quemodectomía izquierda y tres meses después la derecha. La primera fue realizada sin complicaciones. En el abordaje del glomus carotideo derecho se encontró un tumor de $5 \mathrm{~cm}$ que infiltraba la carótida, sin plano de disección (figura 1), y durante la cirugía ocurrió ruptura a nivel de la bifurcación de la carótida, por lo que requirió una resección amplia con derivación carotidea temporal e injerto autólogo de safena invertida. El posoperatorio cursó de forma satisfactoria y sin déficit neurológico. La arteriografía posoperatoria demostró adecuado flujo vascular (figura 2).

El estudio de anatomía patológica reveló un carcinoma de glomus carotideo, con cero de ocho ganglios metastásicos positivos. La paciente recibió radioterapia haz externo convencional posoperatoria y tras 20 años de seguimiento continua sin actividad tumoral. Se realizó estudio y cirugía de resección de los glomus a dos miembros de su familia, sin hallazgos de carcinoma.

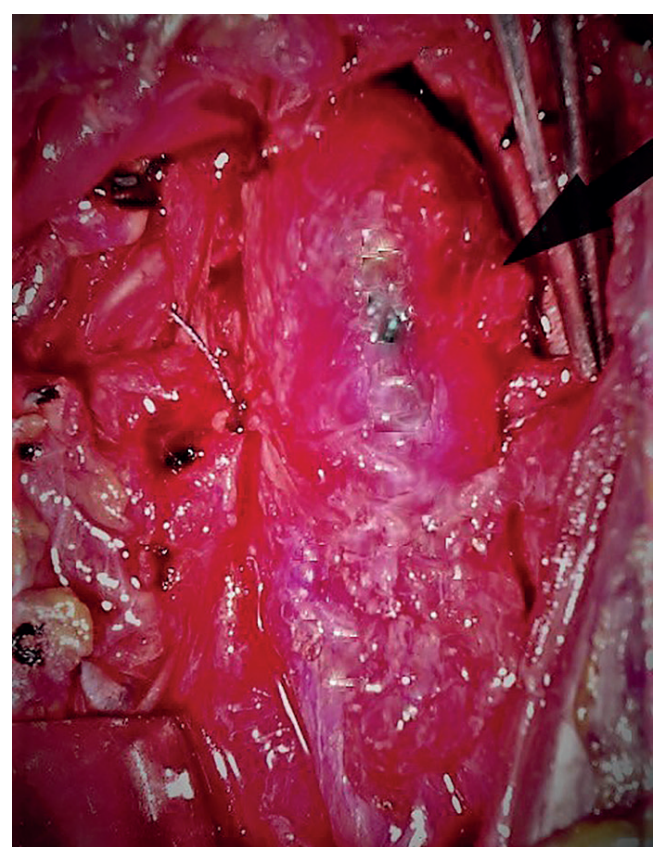

Figura 1. Hallazgo intraoperatorio de la invasión tumoral por el glomus sin plano de clivaje. 


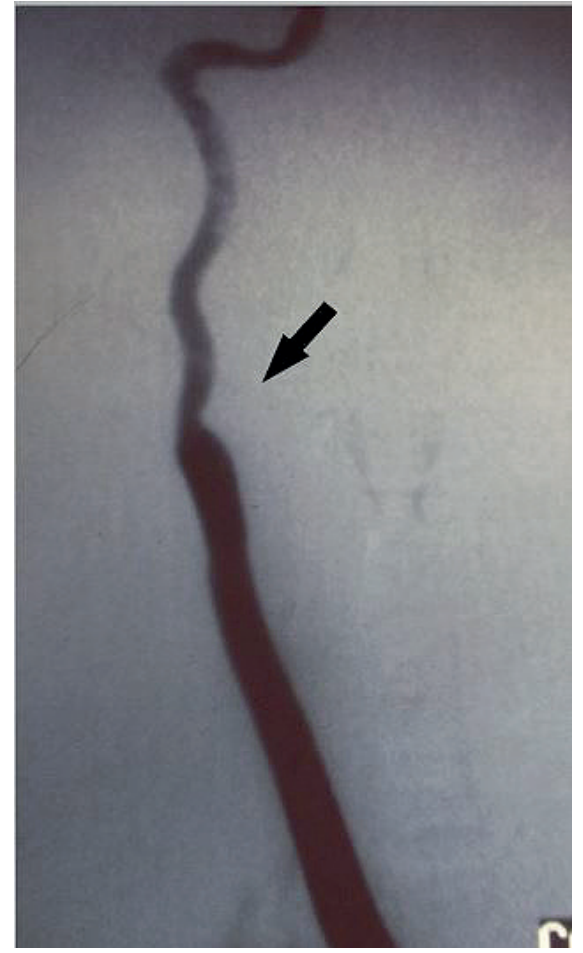

Figura 2. Arteriografía carotidea posoperatoria que muestra el flujo carotideo restituido de la carótida primitiva a la carótida interna con injerto venoso. Ausencia de carótida externa.

\section{Caso 2}

Hombre de 56 años, sin antecedentes de importancia, que consultó por aparición de una masa en el espacio vascular cervical izquierdo, de mínimo crecimiento y sin síntomas asociados. El examen físico de la orofaringe mostró mínima compresión del espacio para-faríngeo y la evaluación del cuello reveló signos de Chevassu y Fontaine presentes, con una masa de $6 \mathrm{~cm}$. Se realizó angiotomografía de vasos cervicales (figuras 3 y 4) que informó un glomus carotideo izquierdo Shamblin III, con posible invasión endoluminal, por lo cual, antes de la cirugía, se realizó un test de oclusión carotidea.

Se procedió a la cirugía practicando resección en bloque del tumor e injerto de PTFE-e-Gore-tex para la reconstrucción carotidea (figura 5) y vaciamiento cervical. La evaluación macroscópica documentó la invasión carotidea por el tumor (figura 6) y la microscópica confirmó un carcino-

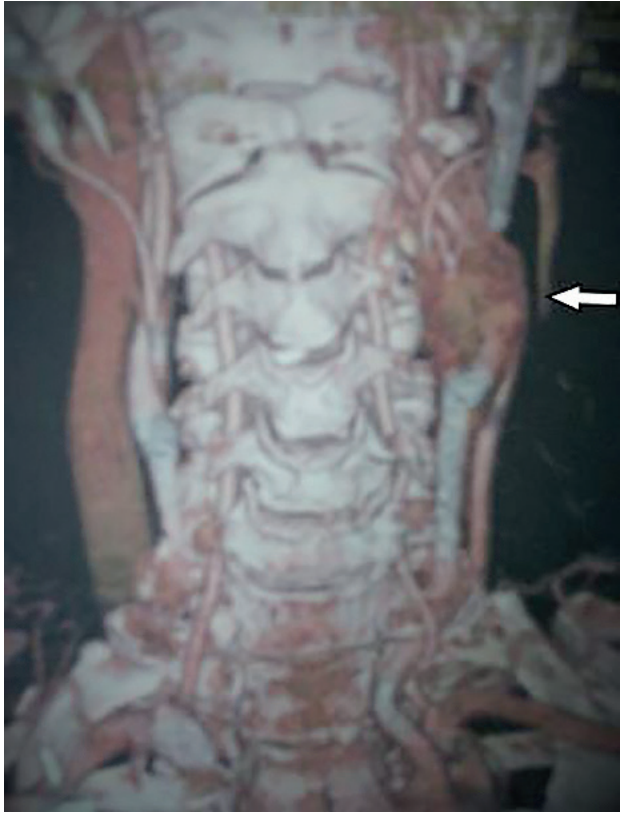

Figura 3. Angio-tomografía de vasos del cuello con reconstrucción en 3D. Se observa el glomus y la pérdida del plano de separación tumor-vaso.

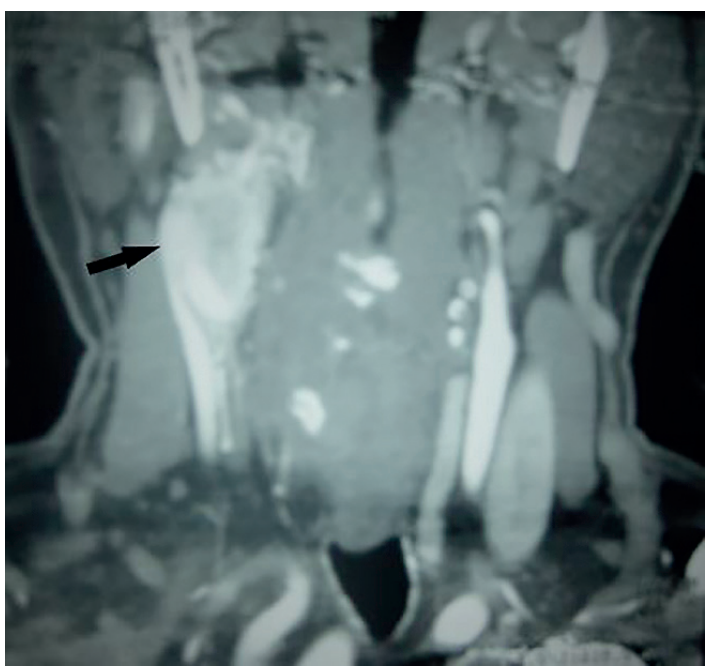

Figura 4. Corte coronal de tomografía contrastada de cuello que sugiere deformidad vascular por invasión en la bifurcación Carotidea.

ma del glomus carotideo metastásico a 12 de 43 ganglios. El paciente evolucionó satisfactoriamente y recibió radioterapia postoperatoria de haz externo 66 GY. Después de un seguimiento de 8 años, se encuentra sin actividad tumoral. 


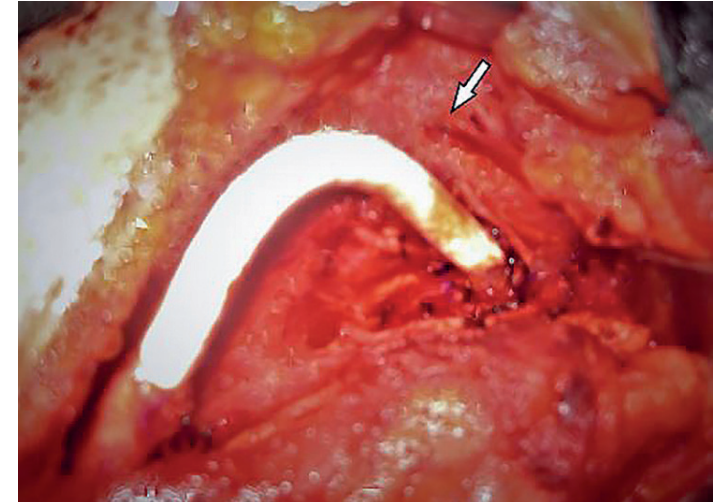

Figura 5. Injerto de Gore-tex implantado entre la carótida primitiva y la carótida interna.

\section{Caso 3}

Paciente de sexo femenino de 61 años, que consultó por masa cervical izquierda de 4 meses de evolución, sin síntomas asociados a la misma y sin antecedentes familiares de importancia. El examen de orofaringe mostró compresión del espacio para-faríngeo y se encontró en el cuello una masa en el espacio vascular izquierdo con signos de Chevassu y Fontaine presentes. La tomografía de cuello con contraste documentó un glomus carotideo Shamblin III (figura 7). La paciente fue intervenida quirúrgicamente sin encontrar un plano de disección y con compromiso ganglionar sospechoso. Se tomó una biopsia del ganglio y se aplazó el tratamiento definitivo. La biopsia confirmó carcinoma del glomus carotideo.

Se procedió a realizar un test de oclusión carotidea y una nueva cirugía, mediante abordaje por mandibulotomía y resección en bloque del tumor, con derivación carotidea intraoperatoria y reconstrucción con injerto de PTFE-e (figura 8), además de vaciamiento de cuello. El posoperatorio fue satisfactorio y la patología confirmó un carcinoma del glomus carotideo metastásico a 1 de 27 ganglios. Recibió radioterapia postquirúrgica IMRT 60 GY, en el seguimiento mediante un Octreoscan se encontraron metástasis hepáticas positivas (figura 9). Recibió somatostatina sistémica con excelente respuesta. $\mathrm{Al}$ año de seguimiento presentaba una enfermedad metastásica estable.

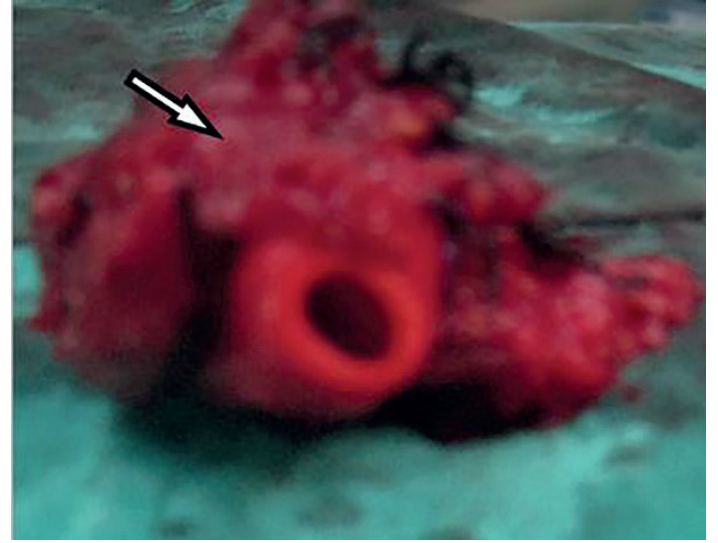

Figura 6. Invasión del tumor a la arteria carótida primitiva.

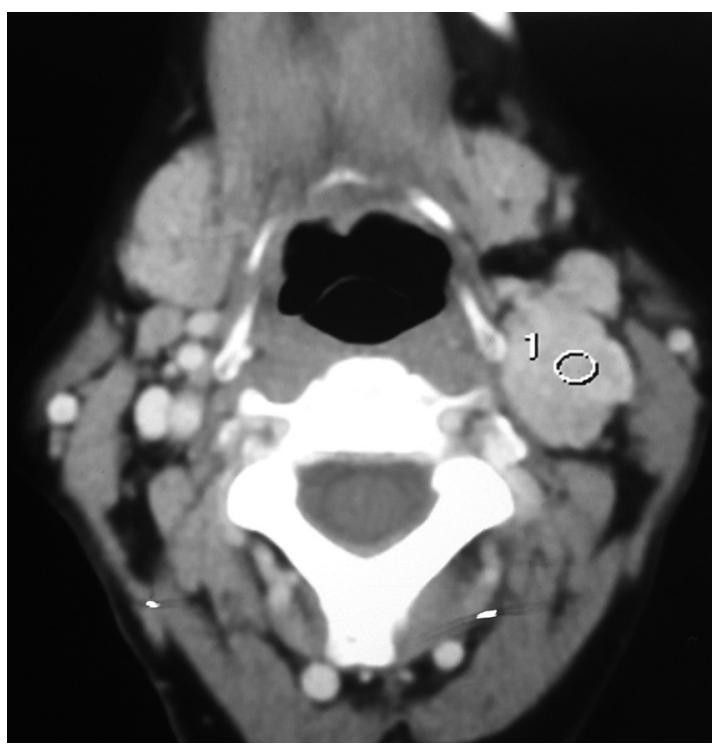

Figura 7. Tomografía axial con contraste, en la que se observa el tumor del glomus carotideo Shamblin III.

\section{Discusión}

\section{Diagnostico preoperatorio}

El glomus carotideo se manifiesta como una masa cervical pulsátil, cuya característica principal es el signo de Fontaine, el cual consiste en el desplazamiento a la palpación del tumor en el plano horizontal más no en el eje axial ${ }^{7}$. También se describe el signo de Chevassu, que consiste en la reducción del volumen del tumor tras la compresión, y el retorno a su tamaño normal luego de 


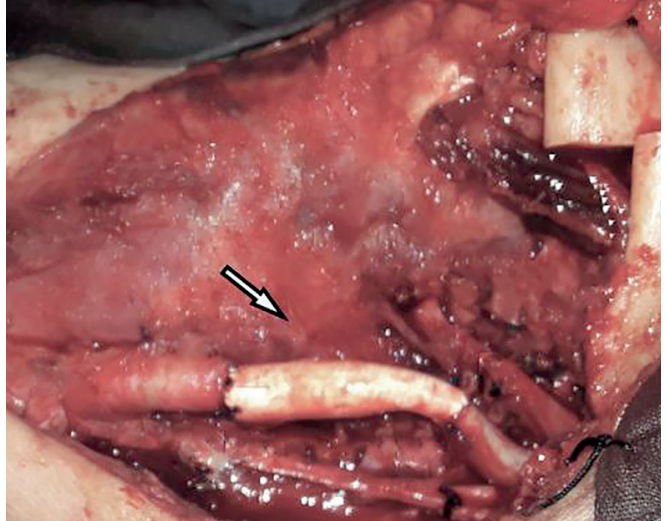

Figura 8. Remplazo arterial con injerto de Gore-tex de la carótida primitiva a la carótida interna.

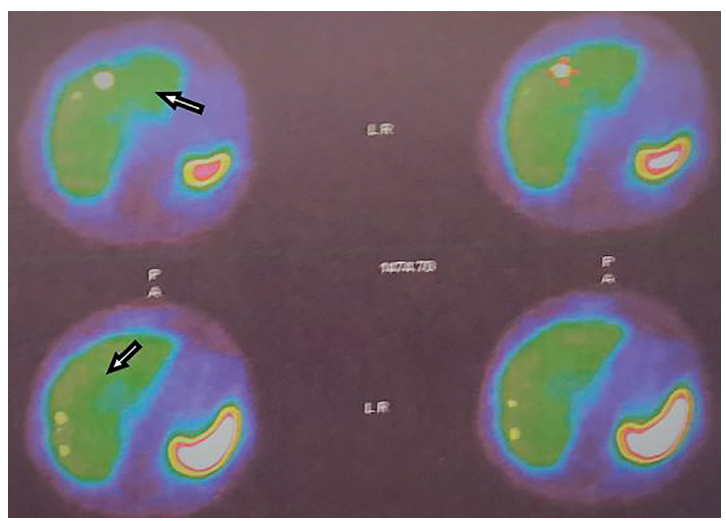

Figura 9. Escanografía (posoperatoria) con octreotido que muestra las lesiones hipercaptantes por las metástasis hepáticas.

unos segundos ${ }^{1}$. Los motivos de consulta pueden ser diversos; múltiples estudios reportan como los más frecuentes la masa cervical de lento crecimiento, seguido de cefalea y sincope ${ }^{7,8}$.

El glomus carotideo maligno (GCM) se presenta como un tumor más firme y grande que el benigno, correspondiendo con mayor frecuencia a la clasificación Shamblin 3 (tumor que rodea completamente las carótidas). Según el estudio realizado por Zhang, estos tumores grandes causan dificultad para palpar la arteria carótida encapsulada, y pueden generar dolor y disfunción de los nervios craneales debido a su capacidad de invadirlos y encapsularlos, comprometiendo principalmente el nervio hipogloso ${ }^{3}$. Los pacien- tes de esta serie presentaron los signos clínicos de glomus carotideo, pero al examen físico no fue posible diferenciar entre malignos o benignos. La incidencia de carcinoma del glomus carotideo de esta serie fue de $2,2 \%$, similar a la reportada en la literatura.

Hasta hace poco tiempo se consideraba a la angiografía carotidea como el estudio imagenológico de elección, en el que se puede observar una masa hipervascularizada asociada al "signo de la lira”, por la separación que ejerce entre la carótida interna y externa. Su principal utilidad es la visualización de la circulación colateral ${ }^{1,9,10}$.

Recientemente se han desarrollado técnicas no invasivas, que se han convertido en una mejor alternativa. El ultrasonido doppler es útil principalmente en el seguimiento posoperatorio, donde la detección temprana de las recurrencias es de suma importancia ${ }^{11}$. La tomografía computarizada (TC) permite estudiar la relación del tumor con las estructuras adyacentes y su extensión a la base del cráneo ${ }^{1}$. En la resonancia nuclear magnética (RNM) se puede identificar la lesión con el signo de "sal y pimienta" en T2 y permite además evaluar la extensión de la lesión ${ }^{8}$. En un estudio multicéntrico reciente, se describió el uso de la TC y de la RNM en 356 pacientes con glomus carotideo, para medir el volumen del tumor y la distancia a la base del cráneo, medidas que asociadas a la clasificación de Shamblin permitieron una mejor predicción de las complicaciones durante la resección (sangrado y lesión de nervios craneales) ${ }^{12}$.

En el presente estudio, solo un paciente tenía hallazgos imagenológicos en la tomografía que sugerían la posibilidad de invasión vascular, lo que alertó acerca de su posible malignidad. La diferenciación entre tumores benignos y malignos antes de la resección no es posible, a no ser que se evidencien metástasis ${ }^{3}$. La presencia de ganglios regionales del nivel II y III, asociada a los glomus carotideos es frecuente, y su sola presencia no se relaciona con malignidad. Se ha descrito la asociación de altos niveles de metaloproteinasas de matriz (especialmente las MMP-1, 2 y 3), en plasma y otros tejidos, con la presentación del $\mathrm{GCM}^{13}$. 


\section{Bilateralidad y presentación familiar}

El glomus carotideo tiene una presentación familiar con herencia autosómica dominante, para la cual se han reportado prevalencias que oscilan entre el $5 \%$ y el $50 \%$. Esta presentación está altamente asociada a mutaciones en los genes que codifican para las subunidades de la succinato deshidrogenasa (SDHB, SDHC y SDHD), lo cual determina el aumento de mediadores de hipoxia intracelular y del factor de crecimiento endotelial vascular, lo que explica la hiperplasia ${ }^{14}$. Entre estas, la mutación SDHB es la que se reporta como más asociada a malignidad, sin embargo, la mutación más común en paragangliomas de cabeza y cuello es la SDHD, la cual no se relaciona con aumento en el riesgo de malignidad ${ }^{15}$.

Rodríguez recomienda realizar tamización clínica y radiológica a los portadores de la mutación SDHD y tamización genética a los demás miembros de la familia, incluyendo a los niños mayores de 10 años ${ }^{14}$. En la paciente con glomus bilateral y familiar, el estudio genético no se realizó por falta de recursos, sin embargo, es evidente el componente genético familiar en 4 hermanos de la misma familia y su descendencia.

Los casos de ocurrencia familiar se presentan usualmente en personas jóvenes (menores de 40 años) con glomus carotideo bilateral ${ }^{16}$. En estos casos se proponen diferentes estrategias de abordaje. Jansen et al., sugieren el manejo conservador o uso de radioterapia en caso de tumores con clasificación Shamblin 3 para evitar la disfunción del nervio craneal o el síndrome baro-reflejo asociado a la resección bilateral ${ }^{17}$. Por otro lado, Lozano propone un algoritmo quirúrgico en las presentaciones bilaterales, según sean los tumores de diferente tamaño, en cuyo caso se haría resección del más pequeño en un primer tiempo, o de tamaño similar, caso en el que se operaría primero el más grande. El abordaje quirúrgico en un segundo tiempo se realizaría a los 2 meses, solo en ausencia de complicaciones de la cirugía previa ${ }^{18}$. Existe consenso en cuanto a la contraindicación del abordaje de las presentaciones bilaterales en un solo tiempo, pues se relaciona con alto riesgo de lesión nerviosa ${ }^{17,18}$. En esta serie no se presentó baro-reflejo en la resección de ambos glomus.

\section{Test de oclusión carotidea}

Actualmente la realización del test de oclusión carotidea (también llamado Matas test), se recomienda como parte del estudio preoperatorio en aquellos casos en los que se considera alto riesgo de lesión vascular intraoperatorio, lo que correspondería a tumores con clasificación Shamblin III ${ }^{3,10}$. Ante la sospecha preoperatoria de carcinoma, sería mandatorio realizar el test de oclusión dada la alta probabilidad de requerir una resección en bloque, con resección y reconstrucción de la arteria.

En nuestro grupo, el test de oclusión carotidea no se realiza de manera rutinaria ni aun en los glomus de gran tamaño, salvo sospecha preoperatoria de invasión o en aquellos casos recurrentes. En los casos de sospecha intraoperatoria de invasión, usualmente relacionados con la imposibilidad de disección sub-adventicia segura o compromiso invasivo de tejidos adyacentes y ante la necesidad de una posible resección carotidea, se recomienda posponer la misma, en espera del test, especialmente para poder tomar decisiones en conjunto con el paciente y su familia, como ocurrió en uno de los casos presentados.

\section{Embolización preoperatoria}

La embolización preoperatoria del glomus carotideo por medio de angiografía fue una práctica ampliamente extendida, no obstante, actualmente es muy controvertida. Esta se justifica por la disminución del flujo sanguíneo al tumor, el cual puede ser hasta del $75 \%$, lo que merma el riesgo de sangrado intraoperatorio o lesión de estructuras adyacentes al mejorar la visualización de estas, y elimina la necesidad de ocluir la arteria carótida ${ }^{10,19}$. No obstante, hasta en el $13 \%$ de los casos, se han descrito complicaciones como necrosis de piel facial y cervical, isquemia o hemorragia a nivel central, disección de la bifurcación carotidea, e incluso, muerte ${ }^{7,10}$.

Además, la fibrosis inflamatoria posembolización dificulta la disección del plano sub-adventicio y no se ha demostrado que esta intervención reduzca significativamente la tasa de complicaciones neurológicas, especialmente la lesión de nervios craneales ${ }^{20}$.

Debido a lo anterior, se considera que la embolización preoperatoria está indicada en casos 
específicos, como identificación de un tumor altamente vascularizado, voluminoso o muy adherido, seguida de la resección inmediata del tumor o hasta 3 días después ${ }^{10,20}$. Zhang et al., recomiendan evaluar previamente la relación entre la arteria que irriga el tumor y el sistema de la arteria carótida interna para evitar el embolismo ectópico

${ }^{20}$. En nuestros pacientes no es rutinaria la embolización, ni aun en casos complejos, dado que en los pocos pacientes que se realizó embolización, la disección en medio de la fibrosis fue más difícil.

\section{Abordaje quirúrgico}

La resección quirúrgica es el tratamiento de elección en el glomus carotideo. Para el GCM también se acepta como el tratamiento de elección, seguida de radioterapia y, eventualmente, quimioterapia según la presencia de tumor residual, recurrencia o metástasis ${ }^{3,10}$. No obstante, se describen algunas situaciones en las que el abordaje quirúrgico está contraindicado, como en caso de disfunción de nervios craneales o del tronco simpático contralateral, paciente anciano frágil, tumores Shamblin III que prevean alta morbilidad asociada y complicaciones isquémicas ${ }^{7,18}$. La posibilidad del síndrome baro-reflejo posterior a la resección del segundo glomus carotideo en los casos de bilateralidad debe ser considerada y discutida con el paciente y su familia.

Existen diversas técnicas quirúrgicas para la resección del glomus carotideo, la más recomendada es la disección sub-adventicia descrita por Gordon-Taylor (incluso en el contexto de tumores Shamblin III con compromiso total de la arteria carótida interna), en la cual el abordaje se realiza desde el espacio avascular entre las carótidas y el tumor, liberando primero la carótida interna, luego disecando la extensión inferior a la bifurcación si está presente, posteriormente liberando la arteria carótida externa y por ultimo exponiéndose y liberando la bifurcación carotidea ${ }^{7,10,21,22}$.

La técnica retro-carotidea también se ha descrito en el abordaje de estos tumores, especialmente en los malignos, implicando la resección de la arteria carótida externa con posterior reconstrucción, usualmente usando injertos de vena safena. Hinojosa describe en dos pacientes con GCM, un abordaje esta técnica, con lo que reportan mejor exposición del plano avascular, reducción del tiempo operatorio y menor pérdida de sangre ${ }^{23}$.

Jansen et al., en su metaanálisis respecto al manejo quirúrgico del glomus carotideo, analizan el riesgo de lesión del nervio craneal, la recurrencia y el abordaje quirúrgico según la clasificación Shamblin. Para los tumores grandes, con clasificación Shamblin III, describen alto riesgo de morbilidad en el abordaje quirúrgico, especialmente si se realiza manipulación de la arteria carótida interna o si se liga la arteria carótida externa. Debido a esto recomiendan realizar la resección únicamente en pacientes con alta morbilidad preoperatoria generada por el tumor, en quienes el riesgo sería menor que el beneficio ${ }^{17}$.

En casos de grandes tumores Shamblin III con alta morbilidad, se sugieren alternativas como la radioterapia o el desempaquetamiento del tumor, manteniendo la disección en márgenes de seguridad para no causar lesión de estructuras adyacentes. Se debe hacer seguimiento del tumor residual, el cual puede ser sometido a radioterapia si aumenta de tamaño ${ }^{17}$. En dos casos de nuestra serie se realizó resección en bloque y reconstrucción arterial con relativa confianza, gracias a la prueba de oclusión satisfactoria realizada preoperatoriamente. En uno de los casos, la disección en bloque se realizó con ayuda de un puente carotideo, sin secuelas neurológicas, pero la disección sub-adventicia no fue posible en ningún caso y siempre culminó en resección en bloque.

Los glomus carotideos malignos, más que causar metástasis a distancia, comprometen los ganglios linfáticos regionales, por lo que durante la resección quirúrgica se recomienda la toma de muestras de ganglios regionales, para realizar estudios por congelación, que de ser positivos hacen mandatoria la adecuada disección ganglionar, especialmente en los niveles II a IV ${ }^{4}$.

\section{Radioterapia}

En años recientes, diferentes estudios han propuesto la radioterapia como abordaje alterno al quirúrgico, describiéndose un control similar del 
tumor y disminución de la morbilidad ${ }^{16-18}$. Usualmente se utiliza la radioterapia convencional a 45 Gy en 25 fracciones ${ }^{16}$. Esta alternativa terapéutica se considera en casos de un tumor grande Shamblin III, que por su extensión sea irresecable, en caso de tumor residual, en paciente anciano frágil, en presencia de lesiones metastásicas irresecables o malas condiciones para realizar cirugía ${ }^{10}$. Se recomienda la adyuvancia en los tumores después de resección del glomus o de la disección de cuello, ante ganglios positivos, como en los casos presentados. Se describen múltiples complicaciones a largo plazo luego de realizar radioterapia, tales como daño vascular, malignidad inducida por radiación y aterosclerosis de la arteria carótida ${ }^{17}$.

\section{Tratamiento de las metástasis}

Las metástasis del GCM se dan usualmente en nodos linfáticos regionales, no obstante, éstas pueden aparecer también a distancia en pulmón, tejido óseo, hígado, páncreas, tiroides, riñón e incluso en el sistema nervioso central. Ante metástasis regionales, usualmente se opta por la resección quirúrgica con radioterapia adyuvante, mientras que las metástasis a distancia se tratan principalmente con quimioterapia ${ }^{3,24}$.

Diversos agentes quimioterapéuticos son utilizados en estos casos, ya sea en terapias combinadas o en monoterapia, siendo los más usados la vincristina, el cisplatino, y la dacarbazina. Recientemente se ha usado el sunitinib, que es un potente inhibidor de múltiples receptores de tirosin quinasa con efecto antiangiogénico efectivo, no obstante, su mayor beneficio ha sido descrito en el tratamiento de paragangliomas simpáticos. Las combinaciones más descritas son vincristina-cisplatino y dacarbazina-vincristina-ciclofosfamida, ésta última está asociada a una tasa de respuesta global del $37 \%$ en paragangliomas malignos ${ }^{24,25}$.

\section{Supervivencia}

Los indicadores pronósticos han sido difíciles de dilucidar. La supervivencia en pacientes con GCM varía entre 1 y 25 años ${ }^{16}$. Sethi et al., desarrollaron un estudio de cohorte retrospectivo, con una muestra de 86 pacientes con paraganglioma maligno de cabeza y cuello, de los cuales el $43 \%$ cursaba con GCM. Analizaron la sobrevida a 5 años en esta cohorte, hallando mejor supervivencia en pacientes tratados únicamente con resección quirúrgica, sólo con metástasis regionales, con paraganglioma maligno en cuerpo carotídeo y menores de 50 años. De estos factores, los estadísticamente significativos para predecir la supervivencia a 5 años fueron el estadio de las metástasis y la edad ${ }^{26}$.

Se han propuesto factores de mal pronóstico para GCM, como el patrón familiar, la mutación SDHB y el tumor múltiple. Lozano et al., proponen seguimiento anual de por vida para estos pacientes ${ }^{18}$. Los pacientes de nuestro grupo permanecen vivos, dos libres de enfermedad y una con enfermedad metastásica estable.

\section{Conclusiones}

El carcinoma del glomus carotideo es poco frecuente y como tal no hay guías claras para el diagnóstico y manejo. El diagnóstico diferencial preoperatorio entre glomus benigno y maligno es difícil, por lo cual debe hacerse una adecuada historia clínica, además de examen físico en busca de factores o hallazgos clínicos de riesgo que puedan sugerir malignidad.

Las imágenes pocas veces muestran compromiso y características de invasión vascular, sin embargo, un hallazgo sugestivo de malignidad debe llevar a realizar otros estudios como el test de oclusión carotidea, que permita planear una cirugía, en la que pueda llegar a ser necesaria la resección en bloque del tumor.

En caso de una disección inusualmente difícil debida a invasión, se debe sospechar malignidad, y se recomienda solo tomar una biopsia de la masa y de los ganglios adyacentes, difiriendo el manejo quirúrgico definitivo.

Siempre que sea posible, el cáncer del glomus carotideo debe tratarse quirúrgicamente, dejando la radioterapia, quimioterapia y hormonoterapia, como terapias adyuvantes.

\section{Cumplimiento de normas éticas}

Consentimiento informado: Este estudio es una revisión retrospectiva de una base de datos y las historias clínicas 
de los pacientes incluidos en la serie, por lo que no implica riesgo para los pacientes, y no hay necesidad de consentimiento informado.

\section{Conflicto de interés: Ninguno}

Fuente de financiación: Financiado por los autores.

\section{Contribución de los autores:}

Concepción y diseño del estudio: Andrés Ignacio ChalaGalindo.

Adquisición de datos: Andrés Ignacio Chala-Galindo, Carolina Andrea Albornoz-Garzón, Carlos Eduardo Gómez-Vera.

Análisis e interpretación de datos: Andrés Ignacio ChalaGalindo, Carolina Andrea Albornoz-Garzón, Carlos Eduardo Gómez-Vera.

Redacción del manuscrito: Andrés Ignacio Chala-Galindo, Carolina Andrea Albornoz-Garzón, Carlos Eduardo GómezVera.

Revisión crítica: Andrés Ignacio Chala-Galindo, Carolina Andrea Albornoz-Garzón, Carlos Eduardo Gómez-Vera.

\section{Referencias}

1. Yañez R, Loyola F, Cornejo J. Tumor de cuerpo carotideo. Rev Chil Cir. 2011;63:513-8. https://doi.org/10.4067/S0718-40262011000500013

2. Fielding JW, Hodson EJ, Cheng X, Ferguson DJP, Eckardt L, Adam J, et al. PHD2 inactivation in type I cells drives HIF-2 $\alpha$-dependent multilineage hyperplasia and the formation of paraganglioma-like carotid bodies. J Physiol. 2018;596:4393-412. https://doi.org/10.1113/JP275996

3. Zhang WC, Cheng JP, Li Q, Zhang L, Wang XD, Anniko M. Clinical and pathological analysis of malignant carotid body tumour: A report of nine cases. Acta Oto-Laryngol. 2009;129:1320-5. https://doi.org/10.3109/00016480802660510

4. Roden D, Myssiorek D. Neck management in malignant head and neck paragangliomas. Oper Tech Otolayngol Head Neck Surg. 2016;27:41-6. https://doi.org/10.1016/j.otot.2015.12.009

5. Rinaldo A, Ferlito A, Myssiorek D, Devaney KO. Which paragangliomas of the head and neck have a higher rate of malignancy? Oral Oncology. 2004;40:458-60. https://doi.org/10.1016/j.oraloncology.2003.08.018

6. Shamblin WR, ReMine WH, Sheps SG, Harrison EG. Carotid body tumor (chemodectoma). Clinicopathologic analysis of ninety cases. Am J Surg. 1971;122:732-9.

7. Kaygusuz I, Karlidag T, Keles E, Yalcin S, Yuksel K. Carotid body tumor: Clinical features. J Craniofac Surg. 2015;26:e586-8. https://doi.org/10.1097/SCS.0000000000002003
8. Darouassi Y, Alaoui M, Mliha-Touati M, Maghraoui O AL, En-nouali A, Bouaity B, et al. Carotid body tumors: a case series and review of the literature. Ann Vasc Surg. 2017;43:265-71. https://doi.org/10.1016/j.avsg.2017.03.167

9. Cáceres H, Silva S, Amarilla R, Laconich D, Sosa K. Tumor de glomus carotídeo. Cir. Parag. 2014;38:35-7.

10. Alvo A, Sedano C, de Grazia JA, Soto F, Vergara JI. Paraganglioma del cuerpo carotideo : Revisión bibliográfica. Acta Otorrinolaringol Cir Cabeza y Cuello. 2015;43:43-9. https://doi.org/10.37076/acorl.v43i1.15

11. Valencia-Díaz E, Clavijo-Rodríguez T, Hernández-Seara A, Savigne-Gutiérrez W. Paragangliomas bilaterales del cuerpo carotideo. Revista Cubana de Angiología. 2016;17:89-97.

12. Kim GY, Lawrence PF, Moridzadeh RS, Zimmerman K, Munoz A, Luna-Ortiz K, et al. New predictors of complications in carotid body tumor resection. J Vasc Surg. 2017;65:1673-9. https://doi.org/10.1016/j.jvs.2016.12.124

13. Serra R, Grande R, Gallelli L, Rende P, Scarcello E, Buffone $\mathrm{G}$, et al. Carotid body paragangliomas and matrix metalloproteinases. Ann Vasc Surg. 2014;28:1665-70. https://doi.org/10.1016/j.avsg.2014.03.022

14. Rodrigues R, Almeida M, Carreiro J, Mendes C, Varino J, Marinho A, et al. Nova mutação do gene sdhd em pacientes com paragangliomas do corpo cartídeo. Angiol Cir Vasc. 2018;14:2-5.

15. Enríquez-Vega ME, Muñoz-Paredes JG, Cossío-Zazueta A, Ontiveros-Carlos Y, Pacheco-Pittaluga E, Bizueto-Rosas H. SDHD gene mutation in Mexican population with carotid body tumor. Cir Cir. 2019;86:33-7. https://doi.org/10.24875/cirue.m18000005

16. Cobos-González E, Aragón-López JA, Soria-Céspedes DR, de la Rosa-Abaroa MA, Martínez-de la Vega-Celorio A, Granados-Gracia M, et al. Paraganglioma maligno (múltiple, multicéntrico y metastásico) en una paciente con historia familiar de paraganglioma. Cir Esp. 2015;93:e127-32. https://doi.org/10.1016/j.ciresp.2015.05.004

17. Jansen TTG, Marres HAM, Kaanders JHAM, Kunst HPM. A meta-analysis on the surgical management of paraganglioma of the carotid body per Shamblin class. Clin Otolaryngol. 2018;43:1104-16. https://doi.org/10.1111/coa.13116

18. Lozano Sánchez FS. Indicaciones quirúrgicas en paragangliomas carotídeos. Cambio del paradigma y propuesta de algoritmos. Angiologia. 2017;69:41-7. https://doi.org/10.1016/j.angio.2016.04.016

19. Amato ACM, Ferreira DDG, da Silva FTF, Uemura MA, Stucchi T de 0, Dos Santos RV. Hybrid surgery in excision of a shamblin II glomus tumor. J Vasc Bras. 2019;18:1-7. https://doi.org/10.1590/1677-5449.012218

20. Zhang J, Fan X, Zhen Y, Chen J, Zheng X, Ma B, et al. Impact of preoperative transarterial embolization of 
carotid body tumor: A single center retrospective cohort experience. Int J Surg. 2018;54:48-52.

https://doi.org/10.1016/j.ijsu.2018.04.032

21. Persky M, Tran T. Acquired vascular tumors of the head and neck. Otolaryngol Clin North Am. 2018;51:255-74. https://doi.org/10.1016/j.otc.2017.09.015

22. Kakkos SK, Zampakis P, Lampropoulos GC, Paridis L, Kaplanis C, Bantouna D, et al. Successful resection of a large carotid body tumor masquerading complete encasement of the internal carotid artery on preoperative imaging. Vasc Endovasc Surg. 2018;52:304-8. https://doi.org/10.1177/1538574418761722

23. Hinojosa CA, Anaya-Ayala JE, Olivares-Cruz S, Laparra-Escareno H, Trolle-Silva A, Angeles-Angeles A.
Malignant Shamblin III carotid body tumors resected with use of the retrocarotid dissection technique in 2 patients. Texas Hear Inst J. 2018;45:92-5. https://doi.org/10.14503/thij-16-6142

24. Jeevan DS, Saleh M, LaBagnara M, Neil JA, Hillard VH. Malignant carotid body tumor presenting with myelopathy: case report. J Neurosurg Spine 2016;24:660-3.

25. Afani L, Errihani H, Awada A. Therapeutic management of malignant paragangliomas: An illustrative case and literature review. Pan Afr Med J. 2019;32:1-5.

26. Sethi RV, Sethi RKV, Herr MW, Deschler DG. Malignant head and neck paragangliomas: Treatment efficacy and prognostic indicators. Am J Otolaryngol. 2013;34:431-8. http://dx.doi.org/10.1016/j.amjoto.2013.03.010 